\title{
Group approach to the paraxial propagation of Hermite-Gaussian modes in a parabolic medium
}

\section{S. Cruz y Cruz *, Z. Gress}

Instituto Politécnico Nacional, UPIITA, Av. Instituto Politécnico Nacional 2580, Col. La Laguna Ticomán, C.P. 07360, Ciudad de México, Mexico

\section{H I G H L I G H T S}

- Ladder and shift operators for the Hermite-Gaussian modes are constructed.

- These operators are used to obtain the generators of the dynamical algebras.

- The Barut-Girardello and Perelomov coherent states are determined.

- The uncertainty relations are considered in connection to the beam quality factor.

\section{A R T I C L E I N F O}

\section{Article history:}

Received 3 March 2017

Accepted 30 May 2017

Available online 13 June 2017

\section{Keywords:}

Hermite-Gaussian modes

Dynamical algebras

Coherent states

\begin{abstract}
A B S T R A C T
A group-theoretical approach to the paraxial propagation of Hermite-Gaussian modes based on the factorization method is presented. It is shown that the $s u(1,1)$ and the $s u(2)$ algebras generate the spectrum of propagation constants at any fixed transversal plane. The complete set of HG modes is decomposed into hierarchies that are used to establish the representation spaces of $S U(1,1)$ and $S U(2)$. The corresponding families of generalized coherent states are constructed and the variances of the quadratures and canonical variables are determined.
\end{abstract}

(c) 2017 Elsevier Inc. All rights reserved.

\section{Introduction}

The set of Hermite-Gaussian (HG) modes has been one of the most studied families of paraxial beams. They play an important role in the study of laser resonators and optical waveguides [1] and have a number of applications in particle trapping [2], communications and signal processing [3], micro- and nano-manipulation of matter [4,5] and high resolution imaging [6] among other areas

\footnotetext{
* Corresponding author.

E-mail address: sgcruzc@ipn.mx (S. Cruz y Cruz).
} 
(see also [7] and references quoted therein). Yet, the study of HG modes is far from being completely exhausted. New developments about their properties, generation and applications are currently addressed [8-11]. In the paraxial approximation, where the directions of the normals to the wavefronts are close to the optical axis, the exact wave equation can be reduced to a parabolic-type one, the paraxial wave equation, by neglecting the second derivative of the field amplitude with respect to the longitudinal coordinate. The HG modes form a complete, orthogonal set of transverse modes with rectangular symmetry that span the solution space of this equation in a homogeneous medium [1]. The corresponding solution in generic weakly inhomogeneous media has been considered in [12] by using curvilinear coordinates. In that work the authors are led to the paraxial wave equation for either a homogeneous or a parabolic medium in the particular case of optical systems admitting separation of the transversal variables. Specifically, the transverse quadratic refractive index characteristic of the parabolic medium has deserved a lot of interest because of its self-focusing and exact collimation properties in the paraxial regime. The propagation and diffraction of Gaussian beams in parabolic media have been considered as well [13-15]. Indeed, it can be shown that the HG modes form an orthogonal set of exact solutions to the paraxial wave equation in optical media with quadratic index profiles.

On the other hand, it is well known that the propagation of paraxial light can be mapped to the evolution of a two-dimensional harmonic oscillator. This is due to the formal equivalence between the parabolic wave equation, describing wave propagation in the paraxial regime, and the Schrödinger equation, governing the time-evolution of a quantum particle in an interaction potential [16-18]. In this context, some operator methods, originally addressed to the study of quantum mechanical systems, have been adapted for the description of light propagation in free space as well as in the presence of optical systems. Such techniques include, e.g., group-theoretical approaches [19-22], the density matrix formalism [23] and the coherent state method [23-26]. In particular, the factorization method is an algebraic technique used to solve the eigenvalue problem and to describe the spectral properties of quantum mechanical systems associated with exactly solvable potentials [27-30]. In this method the second order differential Hamiltonian is factorized, up to an additive constant, as the product of two first order differential operators. These factors can be used to construct the basis elements of the dynamical algebra of the system leading to the group approach of the factorization method (see, [31,32]).

In this work we take advantage of the formal equivalence between the Schrödinger and the paraxial wave equations in order to establish a group-theoretical approach, based on the factorization method, to the paraxial propagation of HG beams in a parabolic medium. We find that the Lie algebras $s u(1,1)$ and $s u(2)$ are the generating algebras of the system at each fixed transversal plane. In this way, the complete set of HG modes can be decomposed into a denumerable set of hierarchies, labeled by a discrete parameter, in two different forms. This means that the whole set of transversal modes is covered either by a set of infinite-dimensional representations of $S U(1,1)$ or by a set of finite-dimensional representations of $S U(2)$. Considering these representations we construct different families of generalized coherent states and determine the corresponding variances for the $s u(1,1)$ and $s u(2)$ quadratures as well as for the canonical variables of position and propagation direction, these last in connection to the propagation factors of each family of coherent states.

The material in this article is organized as follows: In Section 2 we summarize the construction of the fundamental Gaussian-type solution to the paraxial wave equation in a parabolic medium. Next, higher order HG modes are determined by means of the factorization method. In Section 3 we define ladder operators for the HG modes and show that they are propagation invariants. In Section 4 we present the group-approach in terms of the generating algebras and construct the corresponding families of coherent states. In Section 5 we summarize our results and present some conclusions.

\section{The complete set of Hermite-Gaussian modes}

\subsection{The fundamental Gaussian mode}

Consider a weakly inhomogeneous medium with a quadratic refractive index profile

$$
n^{2}(r)=n_{0}^{2}\left(1-\frac{\Omega^{2}}{2} r^{2}\right), \quad \Omega^{2} r^{2} \ll 1,
$$


where $r$ is the radial transversal coordinate, $\Omega$ is a constant parameter characterizing the focusing properties of the medium, and $n_{0}$ is the refractive index at the optical axis. The homogeneous case is recovered by properly taking the limit $\Omega \rightarrow 0$. The corresponding paraxial wave equation is given by

$$
\frac{i}{k_{0}} \frac{\partial U}{\partial z}=\left\{-\frac{1}{2 k_{0}^{2} n_{0}}\left(\frac{\partial^{2}}{\partial x^{2}}+\frac{\partial^{2}}{\partial y^{2}}\right)+n_{0} \frac{\Omega^{2}}{2} r^{2}\right\} U=\mathcal{H} U,
$$

with $U=U(\mathbf{r}, z)$ a representative component of the electromagnetic field, $\mathbf{r}=(x, y)$ the transversal position vector and $k_{0}$ the wave number in free space. As the medium is homogeneous in the $z$ direction, we can find solutions to Eq. (2) as (stationary) eigenstates of $\mathcal{H}$ (the guided HG modes), where the corresponding eigenvalues constitute the spectrum of propagation constants. However, we are interested in non stationary Gaussian wavepacket-type solutions of the form $[12,13]$

$$
U(\mathbf{r}, z)=N(z) e^{i S(z) r^{2}},
$$

where the normalization factor $N$ and the coefficient $S$ in the exponent are complex-valued functions of the longitudinal coordinate $z$ fulfilling the set of equations

$$
\begin{aligned}
& \frac{d}{d z}\left(\frac{2}{k_{0} n_{0}} S\right)+\left(\frac{2}{k_{0} n_{0}} S\right)^{2}+\Omega^{2}=0, \\
& \frac{d}{d z} \ln N(z)=-\frac{2}{k_{0} n_{0}} S .
\end{aligned}
$$

Eq. (4) is a complex Riccati equation that can be reduced to the real Ermakov one

$$
\frac{d^{2} w}{d z^{2}}+\Omega^{2} w=\frac{4}{k_{0}^{2} n_{0}^{2} w^{3}}
$$

by writing

$$
\frac{2}{k_{0} n_{0}} S(z)=\frac{1}{R(z)}+\frac{2 i}{k_{0} n_{0} w^{2}(z)}, \quad \frac{1}{R(z)}=\frac{d}{d z} \ln w(z) .
$$

In this way, the function $w(z)$ comprises all the information of the evolution of the wavepacket and is determinant in the description of the propagation processes of the light beam. Actually, the functions $w(z)$ and $R(z)$ turn out to be, respectively, the width of the beam and the radius of curvature of the wavefront. By substituting (7) into (5) we get the normalization factor

$$
N(z)=\frac{N_{0}}{w(z)} e^{-i \chi(z)}, \quad \frac{d}{d z} \chi(z)=\frac{2}{k_{0} n_{0} w^{2}(z)},
$$

where $N_{0}$ is a constant to be fixed and the function $\chi$, known as the Guoy phase, is a cumulative on-axis phase shift experienced by the wavefront in passing through a focal plane [1] which can be interpreted as a geometric phase [22,33].

It is well known that the solution of (6) can be constructed in terms of the solutions of the corresponding linear equation [34,35]

$$
\frac{d^{2} w}{d z^{2}}+\Omega^{2} w=0
$$

Hence, by setting $w(0)=w_{0}$ we have

$$
w(z)=w_{0}\left[\cos ^{2}(\Omega z)+\frac{1}{\left(\Omega z_{R}\right)^{2}} \sin ^{2}(\Omega z)\right]^{1 / 2}, \quad z_{R}=\frac{k_{0} n_{0} w_{0}^{2}}{2} .
$$

The parameter $z_{R}$ is known as the Rayleigh range or collimation distance. The expression for the Gaussian mode is then

$$
U(\mathbf{r}, z)=\frac{N_{0}}{w(z)} e^{-i \chi(z)} e^{\frac{i k_{0} n_{0} r^{2}}{2 R(z)}} e^{-\frac{r^{2}}{w^{2}(z)}}
$$


with

$$
R(z)=\frac{\tan (\Omega z)}{\Omega\left(1-\Omega^{2} z_{R}^{2}\right)}\left[1+\left(\frac{\Omega z_{R}}{\tan (\Omega z)}\right)^{2}\right], \quad \chi(z)=\frac{2}{k_{0} n_{0}} \int^{z} \frac{d t}{w^{2}(t)} .
$$

Observe the oscillating nature of these functions due to the focalization properties of the medium. The beam experiences, at the same time, the wave propagation (spreading) effects and the medium confinement, resulting in a periodically self-reproducing wavepacket of period $\frac{\pi}{\Omega}$ for $\Omega>0$. The Guoy phase shift between two maxima of the beam width is given by $\chi(z)=\arctan \left(\tan (\Omega z) / \Omega z_{R}\right)$. In the limit $\Omega \rightarrow 0$ we obtain the well known width, radius of curvature and Guoy phase shift for a Gaussian beam supported by a homogeneous medium [1]

$$
w(z)=w_{0} \sqrt{1+\left(\frac{z}{z_{R}}\right)^{2}}, \quad R(z)=z\left[1+\left(\frac{z_{R}}{z}\right)^{2}\right],
$$

and $\chi(z)=\arctan \left(z / z_{R}\right)$. In this case $w_{0}$ is the minimum width and is known as the waist radius of the beam. This means that $z=0$ is the focal plane of the system. The beam doubles its cross section at a distance $z=z_{R}$ from the focus $\left(w\left(z_{R}\right)=\sqrt{2} w_{0}\right)$. Within this distance we say that the beam is well collimated since the width remains nearly constant. As the beam propagates out of this region the width increases as a linear function of $z$. On the other hand, in the particular case $\Omega=\frac{1}{z_{R}}$, the beam propagates with constant width $w(z)=w_{0}$ all along the optical axis, the radius of curvature tends to infinity and the Guoy phase becomes proportional to $z$. In any case, the initial width $w_{0}$ and the Rayleigh range $z_{R}$ can be considered as the transversal and longitudinal characteristic distances of the beam respectively.

\subsection{Higher order Hermite-Gaussian modes}

The existence of more general localized wavepacket-type solutions of the paraxial wave equation with parabolic refractive index is well known [12]. Indeed, one can construct solutions of the form

$$
U(\mathbf{r}, z)=\frac{1}{w(z)} e^{\frac{i k_{0} n_{0} r^{2}}{2 R(z)}} e^{-i \beta \chi(z)} F(u, v), \quad u=\frac{\sqrt{2} x}{w(z)}, \quad v=\frac{\sqrt{2} y}{w(z)},
$$

where the function $F(u, v)$ fulfills the stationary Schrödinger-type equation for a two dimensional oscillator

$$
-\left(\frac{\partial^{2}}{\partial u^{2}}+\frac{\partial^{2}}{\partial v^{2}}\right) F+\rho^{2} F=2 \beta F, \quad \rho^{2}=u^{2}+v^{2} .
$$

The square integrability condition in the $(u, v)$ plane must be imposed if localized beams, carrying finite transverse optical power, are to be constructed. In rectangular coordinates, a complete set of solutions of (15) can be determined from the fundamental solution $F_{00}(u, v)$, by the subsequent application of ladder operators (see, e.g., $[22,36])$. Thus

$$
F_{n m}(u, v)=\frac{1}{\sqrt{n ! m !}}\left(a^{+}\right)^{n}\left(b^{+}\right)^{m} F_{00}(u, v), \quad F_{00}(u, v)=\frac{1}{\sqrt{\pi}} e^{-\frac{\rho^{2}}{2}},
$$

where

$$
a^{ \pm}=a^{ \pm}(z)=\frac{1}{\sqrt{2}}\left(\mp \frac{\partial}{\partial u}+u\right), \quad b^{ \pm}=b^{ \pm}(z)=\frac{1}{\sqrt{2}}\left(\mp \frac{\partial}{\partial v}+v\right)
$$

are operators that fulfill the single mode boson algebra

$$
\left[a^{-}, a^{+}\right]=\left[b^{-}, b^{+}\right]=\mathbb{I}, \quad\left[a^{ \pm}, b^{ \pm}\right]=0 .
$$

The application of these ladder operators on the functions $F_{n m}$ yields

$$
\begin{array}{ll}
a^{+} F_{n m}=\sqrt{n+1} F_{n+1, m}, & a^{-} F_{n m}=\sqrt{n} F_{n-1, m}, \\
b^{+} F_{n m}=\sqrt{m+1} F_{n, m+1}, & b^{-} F_{n m}=\sqrt{m} F_{n, m-1} .
\end{array}
$$




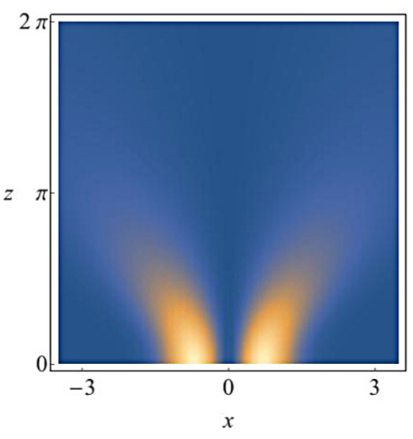

(a) $\Omega=0$.

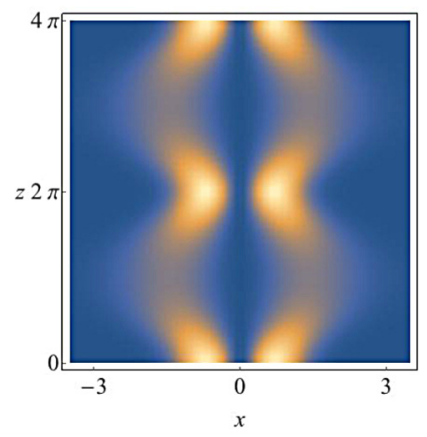

(b) $\Omega=0.5 / z_{R}$.

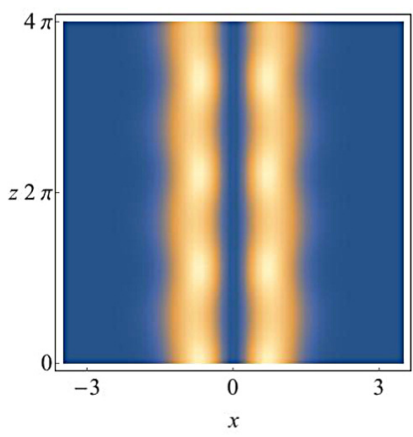

(c) $\Omega=0.9 / z_{R}$.

Fig. 1. Longitudinal plane $y=0$ of the optical intensity distribution for the HG mode $U_{12}(\mathbf{r}, z)$ as it propagates along the $z$ direction, with $\Omega=0$ (a), $\Omega z_{R}=0.5$ (b) and $\Omega z_{R}=0.9$ (c). The transversal and longitudinal coordinates are measured in units of $w_{0}$ and $z_{R}$ respectively.

The solution of the eigenvalue problem (15) takes the form

$$
F_{n m}(u, v)=\frac{1}{\sqrt{\pi 2^{n+m} n ! m !}} e^{-\frac{1}{2} \rho^{2}} H_{n}(u) H_{m}(v), \quad \beta_{n m}=n+m+1,
$$

leading to the well known field amplitude of the HG mode

$$
U_{n m}(\mathbf{r}, z)=\frac{1}{\sqrt{\pi 2^{n+m} n ! m !}} \frac{1}{w(z)} e^{-i(n+m+1) \chi(z)} e^{\frac{i k_{0} n_{0} r^{2}}{2 R(z)}} e^{-\frac{r^{2}}{w^{2}(z)}} H_{n}(u) H_{m}(v),
$$

which is normalized in such a way that the total transverse optical power satisfies $P_{0}=\int_{\mathbb{R}^{2}}\left|U_{n m}(\mathbf{r}, z)\right|^{2}$ $d a=1$, with $d a=d x d y$ the differential element of area in the transversal plane. Notice that the lowest order HG mode $U_{00}(\mathbf{r}, z)$ coincides with the fundamental Gaussian mode (11). In Fig. 1 is shown the longitudinal plane $y=0$ of the optical intensity distribution for the HG mode $U_{12}(\mathbf{r}, z)$ as it propagates in the $z$-direction. For $\Omega=0$ (a), the wavepacket spreads as $z$ increases due to the propagation effects of the beam. On the other hand, for $\Omega=\frac{0.5}{z_{R}}$ (b), the beam focuses itself with a period $2 \pi z_{R}$. The beam becomes well collimated as $\Omega \rightarrow \frac{1}{z_{R}}$ (c).

\section{Ladder operators of Hermite-Gaussian modes}

It turns out that the HG modes can be generated from the fundamental one $U_{00}(\mathbf{r}, z)$ by the subsequent application of ladder operators [25]. Indeed, using (16) the HG mode (22) can be rewritten in the form

$$
U_{n m}(\mathbf{r}, z)=\frac{1}{\sqrt{n ! m !}} e^{-i(n+m) \chi(z)} e^{\frac{i k_{0} n_{0} r^{2}}{2 R(z)}}\left(a^{+}\right)^{n}\left(b^{+}\right)^{m} e^{\frac{-i k_{0} n_{0} r^{2}}{2 R(z)}} U_{00}(\mathbf{r}, z) .
$$

This suggests the introduction of the operators

$$
\begin{array}{ll}
A^{+}=e^{-i \chi} e^{\frac{i k_{0} n_{0} x^{2}}{2 R(z)}} a^{+} e^{\frac{-i k_{0} n_{0} x^{2}}{2 R(z)}}, & A^{-}=\left(A^{+}\right)^{\dagger} \\
B^{+}=e^{-i \chi} e^{\frac{i k_{0} n_{0} y^{2}}{2 R(z)}} b^{+} e^{\frac{-i k_{0} n_{0} y^{2}}{2 R(z)}}, & B^{-}=\left(B^{+}\right)^{\dagger}
\end{array}
$$

which are such that

$$
U_{n m}(\mathbf{r}, z)=\frac{1}{\sqrt{n ! m !}}\left(A^{+}\right)^{n}\left(B^{+}\right)^{m} U_{00}(\mathbf{r}, z) .
$$




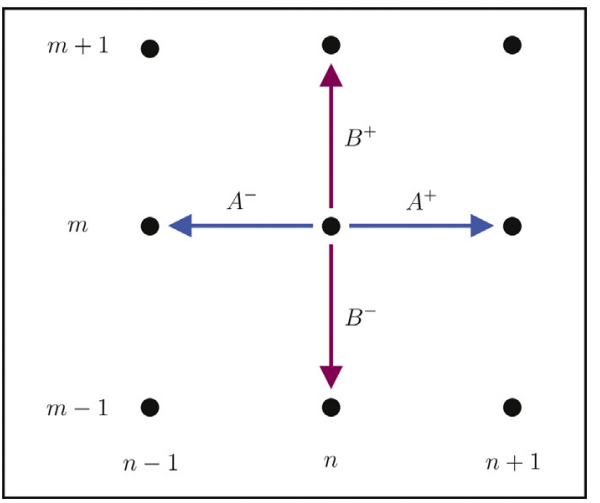

Fig. 2. Action of the boson operators $A^{ \pm}, B^{ \pm}$on the Hermite-Gaussian modes.

The algebraic structure of $A^{ \pm}$and $B^{ \pm}$is inherited from the boson operators $a^{ \pm}, b^{ \pm}$. We have

$$
\begin{aligned}
& {\left[A^{-}, A^{+}\right]=\mathbb{I}, \quad\left[B^{-}, B^{+}\right]=\mathbb{I}, \quad\left[A^{ \pm}, B^{ \pm}\right]=0,} \\
& {\left[N_{x}, A^{ \pm}\right]= \pm A^{ \pm}, \quad\left[N_{y}, B^{ \pm}\right]= \pm B^{ \pm},}
\end{aligned}
$$

with $N_{x}=A^{+} A^{-}$and $N_{y}=B^{+} B^{-}$. Additionally, according to (19)-(20), the action of the operators $A^{ \pm}$, $B^{ \pm}, N_{x}, N_{y}$ on the HG modes (see Fig. 2) produces

$$
\begin{array}{ll}
A^{+} U_{n m}(\mathbf{r}, z)=\sqrt{n+1} U_{n+1, m}(\mathbf{r}, z), & A^{-} U_{n m}(\mathbf{r}, z)=\sqrt{n} U_{n-1, m}(\mathbf{r}, z), \\
B^{+} U_{n m}(\mathbf{r}, z)=\sqrt{m+1} U_{n, m+1}(\mathbf{r}, z), & B^{-} U_{n m}(\mathbf{r}, z)=\sqrt{m} U_{n, m-1}(\mathbf{r}, z), \\
N_{x} U_{n m}(\mathbf{r}, z)=n U_{n m}(\mathbf{r}, z), & N_{y} U_{n m}(\mathbf{r}, z)=m U_{n m}(\mathbf{r}, z) .
\end{array}
$$

It is not difficult to verify that $A^{ \pm}$and $B^{ \pm}$are invariant operators under propagation along the optical axis. In the formal operator description of the paraxial wave optics, the canonical variables of transverse position $\mathbf{r}$ and propagation direction $\mathbf{p}$ are considered as Hermitian operators

$$
\mathbf{r}=(x, y), \quad \mathbf{p}=\left(p_{x}, p_{y}\right)=-\frac{i}{k_{0}}\left(\frac{\partial}{\partial x}, \frac{\partial}{\partial y}\right),
$$

acting on the Hilbert space $\mathcal{L}^{2}\left(\mathbb{R}^{2}\right)$ of square integrable transversal modes of the electromagnetic field $[16,18]$. It is convenient to introduce the vectors $|U(z)\rangle$ to denote the normalized modes of the electromagnetic field. The field amplitude as a function of the position can be expressed as $U(\mathbf{r}, z)=\langle\mathbf{r} \mid U(z)\rangle$. In this way, the paraxial wave equation

$$
\frac{i}{k_{0}} \frac{\partial}{\partial z}|U(z)\rangle=\mathcal{H}|U(z)\rangle, \quad \mathcal{H}=\frac{\mathbf{p}^{2}}{2 n_{0}}+\frac{n_{0}}{2} \Omega^{2} \mathbf{r}^{2},
$$

reveals that the dynamics of the beam is encoded in the family of unitary operators $W\left(z, z_{0}\right)$ describing the propagation process from an initial point $z_{0}$ at the optical axis, to an arbitrary point $z$ in the form

$$
|U(z)\rangle=W\left(z, z_{0}\right)\left|U\left(z_{0}\right)\right\rangle .
$$

As the Hamiltonian does not depend explicitly on the longitudinal coordinate, we have $W\left(z, z_{0}\right)=$ $e^{-i k_{0} \mathcal{H}\left(z-z_{0}\right)}$. This means that $\mathcal{H}$ is the generator of the propagation processes of the light beam along 
the optical axis. Thus, by choosing $z_{0}=0$, the HG mode $\left|U_{n m}(z)\right\rangle$ at an arbitrary point $z$ in the optical axis can be constructed as the unitary transformation

$$
\left|U_{n m}(z)\right\rangle=W(z, 0)\left|U_{n m}(0)\right\rangle .
$$

Moreover, the boson operators $A^{ \pm}, B^{ \pm}$are the evolved versions of the harmonic oscillator ladder operators $a^{ \pm}(0)$ and $b^{ \pm}(0)$ since

$$
A^{ \pm}=e^{-i k_{0} \mathcal{H z}} a^{ \pm}(0) e^{i k_{0} \mathcal{H} z}, \quad B^{ \pm}=e^{-i k_{0} \mathcal{H} z} b^{ \pm}(0) e^{i k_{0} \mathcal{H} z} .
$$

The above expressions are useful to show that $A^{ \pm}, B^{ \pm}$and $H=N_{x}+N_{y}+\mathbb{I}$ are constants of motion as they satisfy the condition

$$
\frac{d \mathcal{O}}{d z}=-i k_{0}[\mathcal{O}, \mathcal{H}]+\frac{\partial \mathcal{O}}{\partial z}=0
$$

In terms of the canonical variables $(\mathbf{r}, \mathbf{p})$ these operators are given by

$$
\begin{aligned}
& A^{+}=-i e^{-i \chi} w\left[\frac{k_{0} p_{x}}{2}-\bar{S} x\right], \quad A^{-}=i e^{i \chi} w\left[\frac{k_{0} p_{x}}{2}-S x\right], \\
& B^{+}=-i e^{-i \chi} w\left[\frac{k_{0} p_{y}}{2}-\bar{S} y\right], \quad B^{-}=i e^{i \chi} w\left[\frac{k_{0} p_{y}}{2}-S y\right], \\
& H=k_{0} z_{R} \frac{w^{2}}{w_{0}^{2}}\left[\frac{\mathbf{p}^{2}}{2 n_{0}}-\frac{1}{k_{0} n_{0}}(S \mathbf{p} \cdot \mathbf{r}+\bar{S} \mathbf{r} \cdot \mathbf{p})+\frac{2}{k_{0}^{2} n_{0}}|S|^{2} \mathbf{r}^{2}\right]+\mathbb{I},
\end{aligned}
$$

where the bar stands for complex conjugation. In the case $\Omega=0$, the expressions (38) coincide with the creation and annihilation operators reported by Nienhuis and Allen for the HG modes in free space [25]. Those operators have also appeared as the conserved creation and annihilation operators of the harmonic states for the free particle [37], and as generalized (invariant) ladder operators of the parametric harmonic oscillator [38].

\section{Mode field hierarchies and generalized coherent states}

In the (n, $m$ )-plane of the parameters that define the whole set of HG modes (depicted in Fig. 2), the operators $A^{ \pm}$and $B^{ \pm}$induce the first order mappings

$$
A^{ \pm}:(n, m) \rightarrow(n \pm 1, m), \quad B^{ \pm}:(n, m) \rightarrow(n, m \pm 1) .
$$

We say that $A^{ \pm}\left(B^{ \pm}\right)$intertwine the HG modes of $m(n)$ fixed. From Fig. 2 one can note that second order compositions of ladder operators allow to intertwine some other sets of modes. In fact, in the context of the multiphoton representation of $S U(n, m)$ and $S U(n)$ [39-42], the generators of the $s u(1,1)$ and $s u(2)$ algebras can be realized as compositions of two single mode boson operators. In the non-degenerate case, these realizations correspond to the Schwinger representations of those algebras [43] (see also [44]). Accordingly, we can construct irreducible representations of $s u(1,1)$ and $s u(2)$ in terms of the ladder operators $A^{ \pm}, B^{ \pm}$. This fact enables the classification of the complete set of HG modes into hierarchies of two different forms. Namely, those associated to the sets of modes with fixed parameter $|m-n|$, and those corresponding to the sets of definite total mode index $n+m$. Next, we are interested in constructing ladder operators connecting the elements of a given hierarchy. Such operators turn out to be the generators of the corresponding Schwinger representations.

\subsection{Mode fields with fixed parameter $|m-n|$}

Consider the second order compositions

$$
\mathcal{K}_{+}=B^{+} A^{+}, \quad \mathcal{K}_{-}=B^{-} A^{-}, \quad \mathcal{K}_{0}=\frac{1}{2}\left(N_{x}+N_{y}+\mathbb{I}\right)=\frac{1}{2} H .
$$




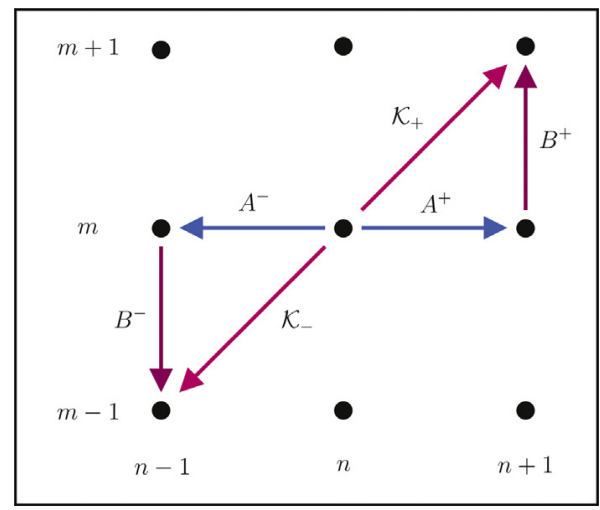

Fig. 3. Action of the operators $\mathcal{K}_{ \pm}$on the Hermite-Gaussian modes. They shift both labels $n$ and $m$ in one unit leaving invariant the quantity $|m-n|$ and thus connecting members of the same $k$-hierarchy.

These operators satisfy the $s u(1,1)$ commutation relations

$$
\left[\mathcal{K}_{-}, \mathcal{K}_{+}\right]=2 \mathcal{K}_{0}, \quad\left[\mathcal{K}_{0}, \mathcal{K}_{ \pm}\right]= \pm \mathcal{K}_{ \pm} .
$$

For the sake of simplicity let us denote the HG mode by the ket $|n, m\rangle$. The operator $\mathcal{K}_{+}\left(\mathcal{K}_{-}\right)$raises (low) both labels $n$ and $m$ in one unit: $\mathcal{K}_{ \pm}:(n, m) \rightarrow(n \pm 1, m \pm 1)$ (see Fig. 3). The HG modes are thus transformed in such a way that the quantity $|m-n|$ is left invariant. Let us define the parameter $k=\frac{1}{2}(|m-n|+1)$. For each fixed $k$, the sets of modes for which $m-n<0$ and $m-n>0$ correspond to two different $k$-hierarchies leading to two realizations of $S U(1,1)$. We say that $\mathcal{K}_{ \pm}$intertwine modes of the same $k$-hierarchy (Fig. 3). Additionally, the Cassimir operator $\mathcal{C}_{2}=\frac{1}{4}\left(N_{x}^{2}+N_{y}^{2}-2 N_{x} N_{y}-\mathbb{I}\right)$ is proportional to the identity: $\mathcal{C}_{2}=k(k-1) \mathbb{I}$, with the Bargman index $k=\frac{1}{2}, 1, \frac{3}{2}, 2, \ldots$. Therefore, the complete mode space decomposes into the direct sum of the set of subspaces $\left\{\mathcal{H}_{+}^{k}, k=\frac{1}{2}, 1, \frac{3}{2}, \ldots, m>n\right\} \cup\left\{\mathcal{H}_{-}^{k}, k=\frac{1}{2}, 1, \frac{3}{2}, \ldots, m<n\right\}$, each one spanned by a particular $k$-hierarchy of HG modes and corresponding to a particular representation of $S U(1,1)$.

If we introduce the parameter $\mu=\frac{1}{2}(m+n+1), \mu=k+n, n=0,1,2, \ldots$ for $m>n$, and $\mu=k+m, m=0,1,2, \ldots$ for $n>m$. Assuming that $m>n$ (the case $m<n$ is easily constructed by interchanging the roles of $n$ and $m), k=\frac{1}{2}(m-n+1)$ and $n=\mu-k, m=\mu+k-1$. It is now convenient to define the ket $|k, \mu\rangle_{\mathcal{K}}$ such that $|n, m\rangle=|\mu-k, \mu+k-1\rangle=|k, \mu\rangle_{\mathcal{K}}$. In this way the action of $\mathcal{K}_{ \pm}, \mathcal{K}_{0}$ on the HG modes can be stated as

$$
\begin{aligned}
\mathcal{K}_{+}|k, \mu\rangle_{\mathcal{K}} & =\sqrt{(\mu+k)(\mu-k+1)}|k, \mu+1\rangle_{\mathcal{K}}, \\
\mathcal{K}_{-}|k, \mu\rangle_{\mathcal{K}} & =\sqrt{(\mu-k)(\mu+k-1)}|k, \mu-1\rangle_{\mathcal{K}}, \quad \mathcal{K}_{-}|k, k\rangle_{\mathcal{K}}=0, \\
\mathcal{K}_{0}|k, \mu\rangle_{\mathcal{K}} & =\mu|k, \mu\rangle_{\mathcal{K}} .
\end{aligned}
$$

\subsection{Barut-Girardello coherent states}

We can construct the generalized Barut-Girardello coherent states $|\xi\rangle_{B G}^{k}$, for each $k$-hierarchy, as eigenmodes of the $S U(1,1)$ annihilation operator $\mathcal{K}_{-}$

$$
\mathcal{K}_{-}|\xi\rangle_{B G}^{k}=\xi|\xi\rangle_{B G}^{k}
$$

with $\xi=|\xi| e^{-i \varphi}$. By using (44) we get

$$
|\xi\rangle_{B G}^{k}=\frac{e^{i k \varphi}}{\sqrt{|\xi| I_{2 k-1}(2|\xi|)}} \sum_{\mu=k}^{\infty} \frac{\xi^{\mu}}{\sqrt{\Gamma(\mu+k) \Gamma(\mu-k+1)}}|k, \mu\rangle_{\mathcal{K}}
$$




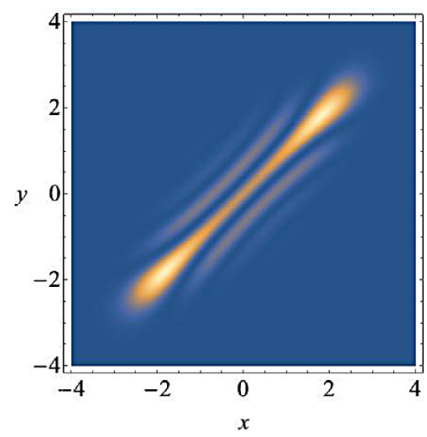

(a) $\varphi=0$.

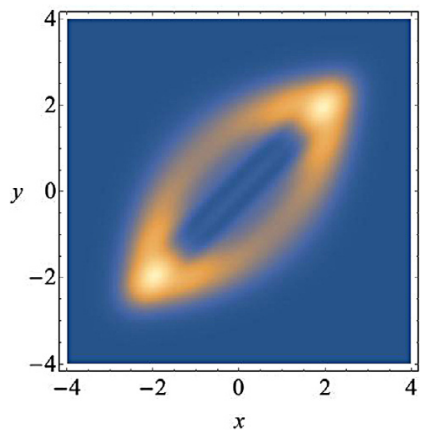

(b) $\varphi=\frac{\pi}{4}$.

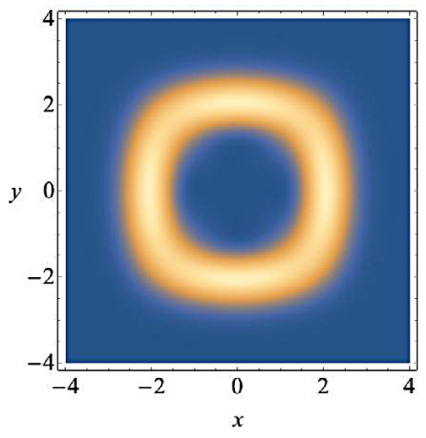

(c) $\varphi=\frac{\pi}{2}$.

Fig. 4. Intensity distributions of the Barut-Girardello coherent states, in the focal plane $z=0$, for $k=\frac{1}{2}$, $|\xi|=5$ and different values of the phase $\varphi$. The variables $x, y$ are measured in units of $w_{0}$.

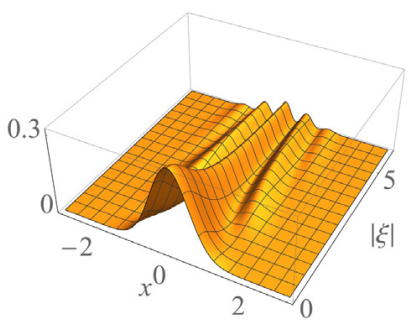

(a) $\varphi=0, k=\frac{1}{2}$.

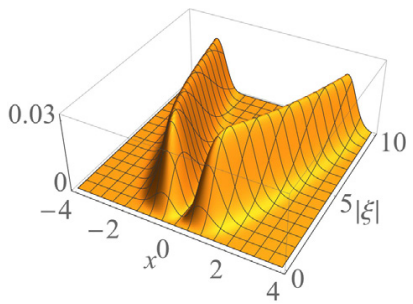

(d) $\varphi=\frac{\pi}{2}, k=2$.

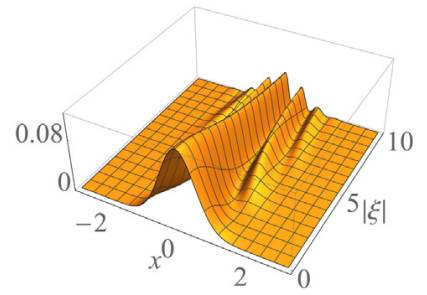

(b) $\varphi=0, k=\frac{5}{2}$.

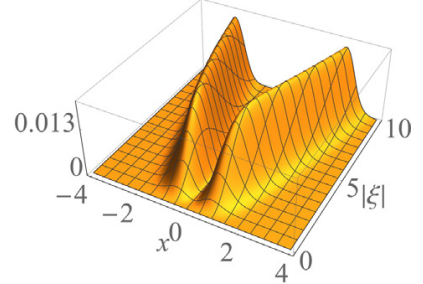

(e) $\varphi=\frac{\pi}{2}, k=5$.

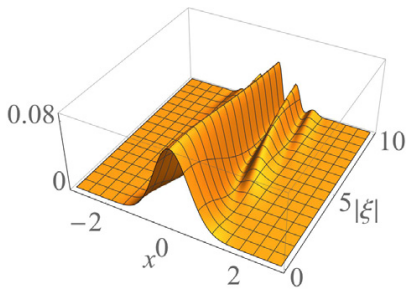

(c) $\varphi=0, k=\frac{9}{2}$.

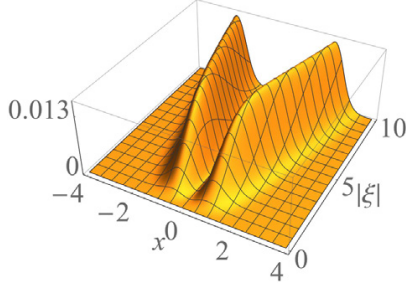

(f) $\varphi=\frac{\pi}{2}, k=8$.

Fig. 5. Intensity distributions of the Barut-Girardello coherent states $U_{B G}^{k}(\mathbf{r}, z)$, as functions of $|\xi|$ at $y=0$, for different values of $k$ and $\varphi$. The transversal variable $x$ is measured in units of $w_{0}$.

where $I_{v}(z)$ is the modified Bessel function of the first kind [45]. The corresponding position representation $U_{B G}^{k}(\mathbf{r}, z)=\langle\mathbf{r} \mid \xi\rangle_{B G}^{k}$ is given by

$$
U_{B G}^{k}(\mathbf{r}, z)=\frac{e^{i k \varphi}}{\sqrt{|\xi| I_{2 k-1}(2|\xi|)}} \sum_{\mu=k}^{\infty} \frac{\xi^{\mu}}{\sqrt{\Gamma(\mu+k) \Gamma(\mu-k+1)}} U_{\mu-k, \mu+k-1}(\mathbf{r}, z) .
$$

In Fig. 4 it is shown the transversal plane $z=0$ of the optical intensity pattern of the coherent states (48) for $k=\frac{1}{2},|\xi|=5$ and different values of $\varphi$. Around the points $\varphi=s \pi, s=0,1,2, \ldots$, these are narrow distributions presenting a series of local maxima according to the value of $|\xi|$. As $\varphi$ approaches integer multiples of $\frac{\pi}{2}$, the wavepackets turn into rectangular patterns centered at the optical axis. Fig. 5, on the other hand, shows the intensity at the longitudinal plane $y=0$, as a function of $|\xi|$, for different values of $k$ and $\varphi$. Note that for $\varphi=0$ the pattern becomes narrower and exhibits more than 
one peak as $|\xi|$ increases. For $\varphi=\frac{\pi}{2}$ the intensity presents two peaks for any value of $|\xi|$ (consistently with Fig. 4(c)) and the wavepacket spreads as $|\xi|$ increases.

In Fig. 6 we present the plane $y=0$ of the distributions $\left|U_{B G}^{k}(\mathbf{r}, z)\right|^{2}$ with $\varphi=\frac{\pi}{2}, k=10$, and different values of $|\xi|$, as the beam propagates along the optical axis. Consistently with Figs. 4(c) and $5(\mathrm{~d}, \mathrm{e}, \mathrm{f})$, they present only two maxima oscillating with an amplitude that increases with $|\xi|$. In these plots we have chosen $\Omega=\frac{0.5}{z_{R}}$ so that beam focuses itself with a period $2 \pi z_{R}$.

\section{Variances and uncertainty relations}

The operators $\mathcal{K}_{ \pm}$define the quadratures [44]

$$
\mathcal{K}_{1}=\frac{1}{2 i}\left(\mathcal{K}_{+}-\mathcal{K}_{-}\right), \quad \mathcal{K}_{2}=-\frac{1}{2}\left(\mathcal{K}_{+}+\mathcal{K}_{-}\right),
$$

that fulfill the commutation relations

$$
\left[\mathcal{K}_{0}, \mathcal{K}_{1}\right]=i \mathcal{K}_{2}, \quad\left[\mathcal{K}_{1}, \mathcal{K}_{2}\right]=-i \mathcal{K}_{0}, \quad\left[\mathcal{K}_{2}, \mathcal{K}_{0}\right]=i \mathcal{K}_{1},
$$

and give rise to the inequality

$$
\Delta \mathcal{K}_{1} \Delta \mathcal{K}_{2} \geq \frac{1}{2}\left|\left\langle\mathcal{K}_{0}\right\rangle\right|
$$

For the Barut-Girardello coherent states (47) we get the variances

$$
\left(\Delta \mathcal{K}_{1}\right)^{2}=\left(\Delta \mathcal{K}_{2}\right)^{2}=\Delta \mathcal{K}_{1} \Delta \mathcal{K}_{2}=\frac{k}{2}+\frac{I_{2 k}(2|\xi|)}{2 I_{2 k-1}(2|\xi|)}|\xi|=\frac{1}{2}\left\langle\mathcal{K}_{0}\right\rangle_{B G}^{k},
$$

meaning that these modes are minimum uncertainty states with average parameter $\bar{\beta}_{k}=2\left\langle\mathcal{K}_{0}\right\rangle_{B G}^{k}$ described by $|\xi|$.

Now, let us consider the variances of the canonical variables r, p. First, the expressions (38)-(39) allow us to write

$$
\begin{aligned}
& x=\frac{w}{2}\left[e^{i \chi} A^{+}+e^{-i \chi} A^{-}\right], \quad y=\frac{w}{2}\left[e^{i \chi} B^{+}+e^{-i \chi} B^{-}\right], \\
& p_{x}=\frac{w}{k_{0}}\left[e^{i \chi} S A^{+}+e^{-i \chi} \bar{S} A^{-}\right], \quad p_{y}=\frac{w}{k_{0}}\left[e^{i \chi} S B^{+}+e^{-i \chi} \bar{S} B^{-}\right] .
\end{aligned}
$$

On the other hand, it is possible to show that

$$
\begin{aligned}
& A^{-}|\xi\rangle_{B G}^{k}=\sqrt{\frac{I_{2 k}(2|\xi|)}{|\xi| I_{2 k-1}(2|\xi|)}} \xi|\xi\rangle_{B G}^{k+\frac{1}{2}}, \\
& B^{-}|\xi\rangle_{B G}^{k}=\sqrt{\frac{I_{2 k-2}(2|\xi|)}{|\xi| I_{2 k-1}(2|\xi|)}}|\xi||\xi\rangle_{B G}^{k-\frac{1}{2}},
\end{aligned}
$$

leading to

$$
\begin{aligned}
& \langle x\rangle_{B G}^{k}=\langle y\rangle_{B G}^{k}=\left\langle p_{x}\right\rangle_{B G}^{k}=\left\langle p_{y}\right\rangle_{B G}^{k}=0, \\
& \left\langle N_{x}\right\rangle_{B G}^{k}=\frac{I_{2 k}(2|\xi|)}{I_{2 k-1}(2|\xi|)}|\xi|, \quad\left\langle N_{y}\right\rangle_{B G}^{k}=\frac{I_{2 k-2}(2|\xi|)}{I_{2 k-1}(2|\xi|)}|\xi| \\
& \left\langle x^{2}\right\rangle_{B G}^{k}=\frac{w^{2}}{4} \eta_{x}, \quad\left\langle p_{x}^{2}\right\rangle_{B G}^{k}=|S|^{2} \frac{w^{2}}{k_{0}^{2}} \eta_{x}, \quad\left\langle\left[x, p_{x}\right]_{+}\right\rangle_{B G}^{k}=\frac{w^{2}}{k_{0}} \operatorname{Re}(S) \eta_{x}, \\
& \left\langle y^{2}\right\rangle_{B G}^{k}=\frac{w^{2}}{4} \eta_{y}, \quad\left\langle p_{y}^{2}\right\rangle_{B G}^{k}=|S|^{2} \frac{w^{2}}{k_{0}^{2}} \eta_{y}, \quad\left\langle\left[y, p_{y}\right]_{+}\right\rangle_{B G}^{k}=\frac{w^{2}}{k_{0}} \operatorname{Re}(S) \eta_{y},
\end{aligned}
$$

where $\eta_{x}=2\left\langle N_{x}\right\rangle_{B G}^{k}+1, \eta_{y}=2\left\langle N_{y}\right\rangle_{B G}^{k}+1$, and the bracket $[A, B]_{+}=A B+B A$ stands for the anticommutator of $A$ and $B$. Therefore, the Schrödinger-Robertson uncertainty relationship takes the form

$$
(\Delta x)^{2}\left(\Delta p_{x}\right)^{2}-\left(\Delta x p_{x}\right)^{2}=\frac{1}{4 k_{0}^{2}}\left[\frac{2 I_{2 k}(2|\xi|)}{I_{2 k-1}(2|\xi|)}|\xi|+1\right] \geq \frac{1}{4 k_{0}^{2}}
$$




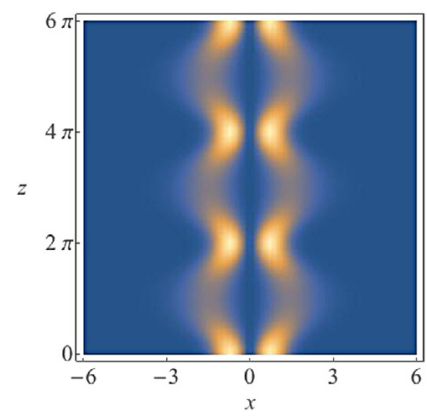

(a) $|\xi|=1$.

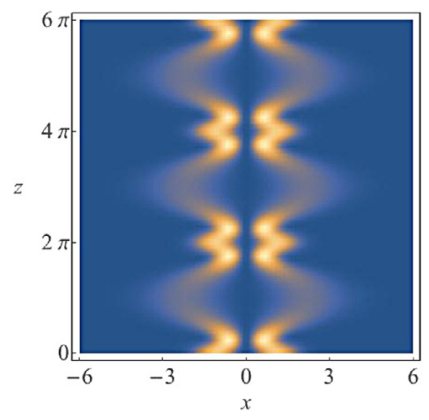

(b) $|\xi|=5$.

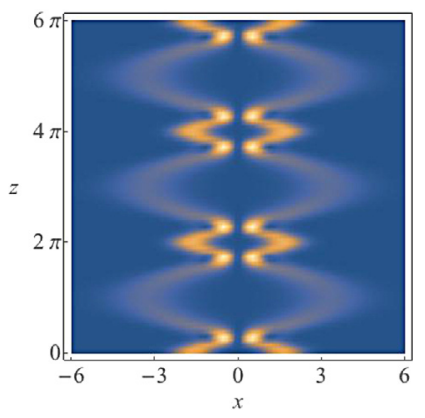

(c) $|\xi|=8$

Fig. 6. Longitudinal plane $y=0$ of the intensity pattern $\left|U_{B G}^{k}(\mathbf{r}, z)\right|^{2}$ with $k=10, \varphi=\frac{\pi}{2}$ and $\Omega=0.5 / z_{R}$. The two maxima of the wavepacket oscillate with an amplitude that increases with $|\xi|$. The transversal and the longitudinal variables are measured in units of $w_{0}$ and $z_{R}$ respectively.

and

$$
(\Delta y)^{2}\left(\Delta p_{y}\right)^{2}-\left(\Delta y p_{y}\right)^{2}=\frac{1}{4 k_{0}^{2}}\left[\frac{2 I_{2 k-2}(2|\xi|)}{I_{2 k-1}(2|\xi|)}|\xi|+1\right] \geq \frac{1}{4 k_{0}^{2}}
$$

with $\Delta(A B)=\frac{1}{2}[A, B]_{+}-\langle A\rangle\langle B\rangle$. As $[45]$

$$
I_{v}(x) \sim \frac{\left(\frac{1}{2} x\right)^{v}}{\Gamma(v+1)}, \text { for } x \rightarrow 0, \quad v \neq-1,-2, \ldots
$$

one can show that the equality holds in (61) for $|\xi|=0$ and any value of $k$, and in (62) for $|\xi|=0$ and $k=\frac{1}{2}$. This asymmetry is the result of the choice $m-n>0$. A converse situation occurs for the Barut-Girardello coherent states associated to the $k$-hierarchy with $m-n<0$.

\section{The propagation factor}

The Schrödinger-Robertson uncertainty relation may be connected with the propagation factor $M^{2}$ of the beam. This is a parameter containing information about the stability of the beam shape under wave propagation effects. Such parameter is defined as the ratio of the "real beam space-beamwidth variances product" to the "ideal Gaussian beam space-beamwidth variances product" [46,47], so that it fulfills the condition $M^{2} \geq 1$. In the case that the first order moments of the canonical variables vanish, the propagation factor can be expressed in terms of the second order moments as $[48,49]$

$$
M^{2}=k_{0} \sqrt{\left\langle\mathbf{r}^{2}\right\rangle\left\langle\mathbf{p}^{2}\right\rangle-\frac{1}{4}\langle\mathbf{r} \cdot \mathbf{p}+\mathbf{p} \cdot \mathbf{r}\rangle^{2}}
$$

Substituting the expressions (58)-(60) into (64), for the Barut-Girardello coherent states we find

$$
M^{2}=1+\frac{I_{2 k}(2|\xi|)+I_{2 k-2}(2|\xi|)}{I_{2 k-1}(2|\xi|)}|\xi| \geq 1
$$

From (63) we can see that $M^{2} \sim 2 k$ as $|\xi| \rightarrow 0$. Additionally, the expression [45]

$$
I_{v}(x) \sim \frac{e^{x}}{\sqrt{2 \pi x}}\left[1-\frac{4 v^{2}-1}{8 x}+\cdots\right], \quad \text { as } \quad|x| \rightarrow \infty,
$$

implies that $M^{2}$ behaves as a linear function of $|\xi|$ for $|\xi| \rightarrow \infty$ (see Fig. 7). The equality sign in (65) is only valid for the coherent state corresponding to $|\xi|=0$ and $k=\frac{1}{2}$ (the fundamental Gaussian mode). Yet, the propagation factor of the HG mode $U_{n m}(\mathbf{r}, z)$ is given by $M_{H G}^{2}=n+m+1=\beta_{n m}$. Therefore, for small values of $|\xi|, M^{2} \sim 2 k=m-n+1<M_{H G}^{2}$, meaning that the Barut-Girardello 


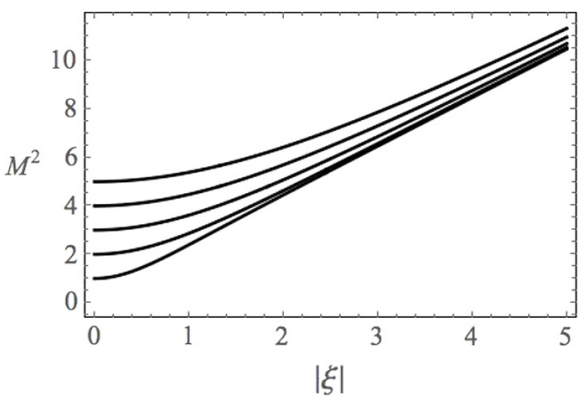

Fig. 7. Propagation factor $M^{2}$ of the Barut-Girardello coherent states for $k=\frac{1}{2}, 1, \frac{3}{2}, 2, \frac{5}{2}$ (lower curves correspond to smaller values of $k$ ). For $|\xi| \sim 0, M^{2} \sim 2 k$. The case $|\xi|=0, k=\frac{1}{2}$ corresponds to the fundamental Gaussian mode.

coherent states are less affected by the diffraction effects than each one of its HG components. For large values of $|\xi|$, however, this is no longer true as $M^{2}$ increases indefinitely.

\section{3. $S U(1,1)$ Perelomov coherent states}

We can also construct generalized coherent states according to the Perelomov approach as displaced versions of the extremal mode $|k, k\rangle_{\mathcal{K}}$

$$
|\xi\rangle_{P}^{k}=D(\xi)|k, k\rangle_{\mathcal{K}}, \quad D(\xi)=e^{\xi \mathcal{K}_{+}-\bar{\xi} \mathcal{K}_{-}} .
$$

As $\mathcal{K}_{ \pm}, \mathcal{K}_{0}$ correspond to the Schwinger representation of $S U(1,1)$, the displacement operator is the non degenerate two-photon squeezing operator $D(\xi)=e^{\xi A^{+} B^{+}-\bar{\xi} A^{-} B^{-}}$. From (44) we can show that

$$
\left(\mathcal{K}_{+}\right)^{s}|k, k\rangle_{\mathcal{K}}=\sqrt{\frac{s ! \Gamma(2 k+s)}{\Gamma(2 k)}}|k, k+s\rangle_{\mathcal{K}} .
$$

In this way, the disentangling formula

$$
D(\xi)=e^{\xi \mathcal{K}_{+}-\bar{\xi} \mathcal{K}_{-}}=e^{\zeta \mathcal{K}_{+}} e^{\ln \left(1-|\zeta|^{2}\right) \mathcal{K}_{0}} e^{-\bar{\zeta} \mathcal{K}_{-}}, \quad \zeta=\frac{\xi}{|\xi|} \tanh |\xi|,
$$

leads us to

$$
|\xi\rangle_{P}^{k}=\frac{\left(1-|\zeta|^{2}\right)^{k}}{\sqrt{\Gamma(2 k)} \zeta^{k}} \sum_{\mu=k}^{\infty} \sqrt{\frac{\Gamma(k+\mu)}{(\mu-k) !}} \zeta^{\mu}|k, \mu\rangle_{\mathcal{K}}
$$

The corresponding position representation is given by

$$
U_{P}^{k}(\mathbf{r}, z)=\langle\mathbf{r} \mid \xi\rangle_{P}^{k}=\frac{\left(1-|\zeta|^{2}\right)^{k}}{\sqrt{\Gamma(2 k)} \zeta^{k}} \sum_{\mu=k}^{\infty} \sqrt{\frac{\Gamma(k+\mu)}{(\mu-k) !}} \zeta^{\mu} U_{\mu-k, \mu+k-1}(\mathbf{r}, z)
$$

(compare to [36,44]). In Fig. 8 we present the plots of $\left|U_{P}^{k}(\mathbf{r}, z)\right|^{2}$ for $k=\frac{5}{2},|\zeta|=0.75$ and different values of $\varphi$. The intensity pattern in this case contains $2 k$ narrow peaks at $\varphi=s \pi$, with $s=0,1,2, \ldots$, and evolves into a rectangular distribution as $\varphi$ approaches odd multiples of $\frac{\pi}{2}$. Fig. 9, in turn, shows the optical intensity as a function of $|\zeta|$, for $y=0$ and different values of $k$ and $\varphi$. For $\varphi=0$ the distribution becomes narrower for larger values of $|\zeta|$. The latter means that the wavepacket becomes more localized for this particular value of $y$. For $\varphi \neq 0$ the light beam spreads as $|\zeta|$ takes larger values. The propagation of the wavepacket along the optical axis, at the longitudinal plane $y=0$, is presented in Fig. 10. Notice the oscillating pattern due to the focusing properties of the medium: as the parameter $\Omega=\frac{0.5}{z_{R}}$, the beam focuses itself with a period $2 \pi z_{R}$. The amplitude of the oscillations increases with $|\zeta|$ (see also Fig. 9(f)). 


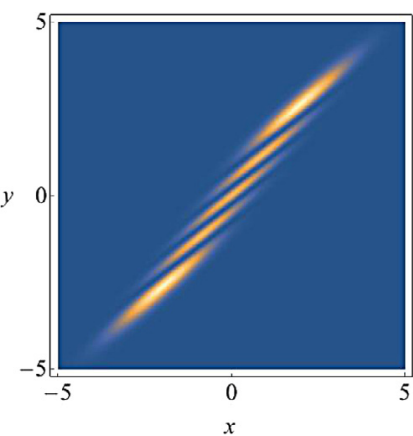

(a) $\varphi=0$.

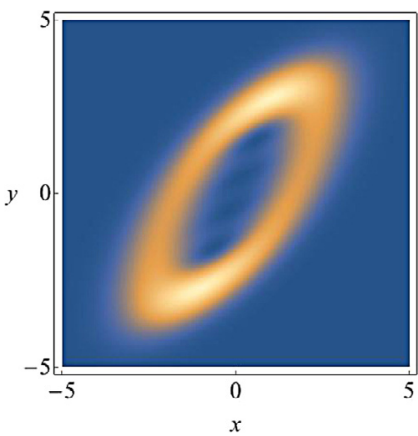

(b) $\varphi=\frac{\pi}{4}$.

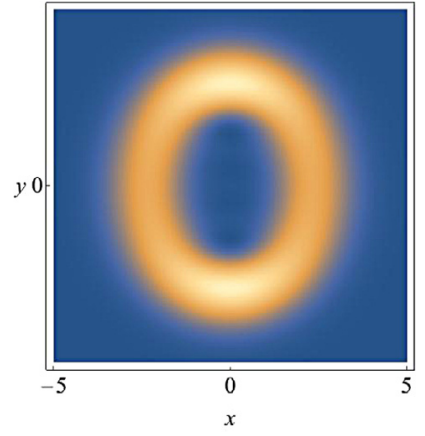

(c) $\varphi=\frac{\pi}{2}$.

Fig. 8. Transversal intensity distributions of the $S U(1,1)$ Perelomov coherent states at the focal plane $z=0$, for $|\zeta|=0.75$, $k=\frac{5}{2}$ and different values of the phase $\varphi$. The pattern consists of 5 narrow peaks at $\varphi=0$ evolving into a rectangular distribution as $\varphi$ approaches $\frac{\pi}{2}$. The transversal variables $x, y$ are measured in units of $w_{0}$.

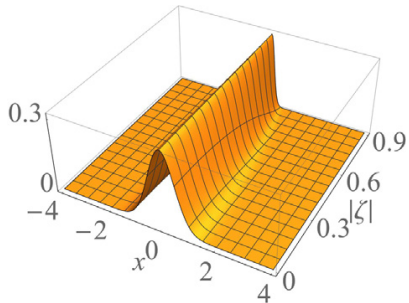

(a) $\varphi=0, k=\frac{1}{2}$.

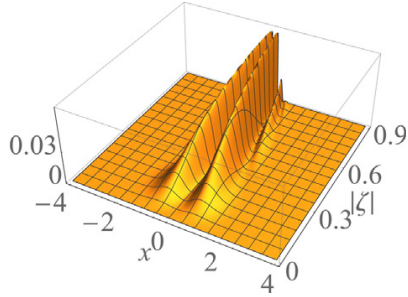

(d) $\varphi=0, k=5$.

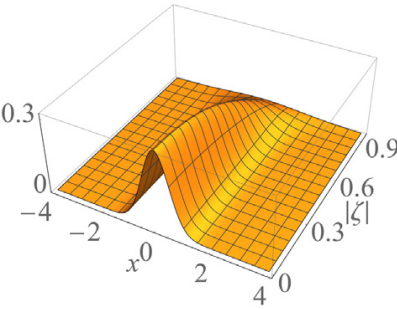

(b) $\varphi=\frac{\pi}{4}, k=\frac{1}{2}$.

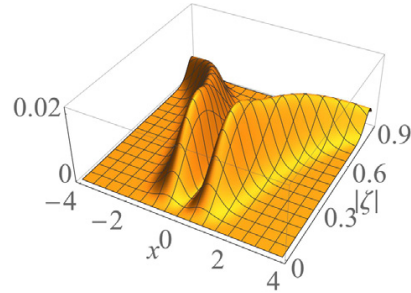

(e) $\varphi=\frac{\pi}{4}, k=5$.

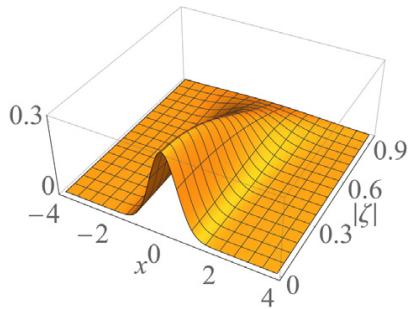

(c) $\varphi=\frac{\pi}{2}, k=\frac{1}{2}$.

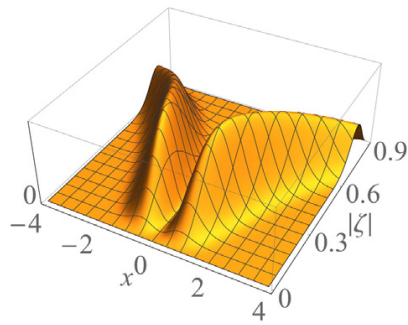

(f) $\varphi=\frac{\pi}{2}, k=5$.

Fig. 9. Optical intensity patterns of the Perelomov coherent states as function of $|\zeta|$ for $y=0$ and the values of $k$ and $\varphi$ indicated in each case. In this longitudinal plane the pattern becomes narrower for larger values of $|\zeta|$ at $\varphi=0$. For $\varphi \neq 0$, the wavepacket spreads as $|\zeta|$ grows. The transverse variable $x$ is measured in units of $w_{0}$.

\section{Variances and squeezing}

The uncertainties of the $S U(1,1)$ variables for these modes are given by

$$
\begin{aligned}
& \left(\Delta \mathcal{K}_{1}\right)^{2}=\frac{1}{2}\left[1+\left(\frac{2 \operatorname{Im}(\zeta)}{1-|\zeta|^{2}}\right)^{2}\right]\left\langle\mathcal{K}_{0}\right\rangle_{0}, \\
& \left(\Delta \mathcal{K}_{2}\right)^{2}=\frac{1}{2}\left[1+\left(\frac{2 \operatorname{Re}(\zeta)}{1-|\zeta|^{2}}\right)^{2}\right]\left\langle\mathcal{K}_{0}\right\rangle_{0},
\end{aligned}
$$




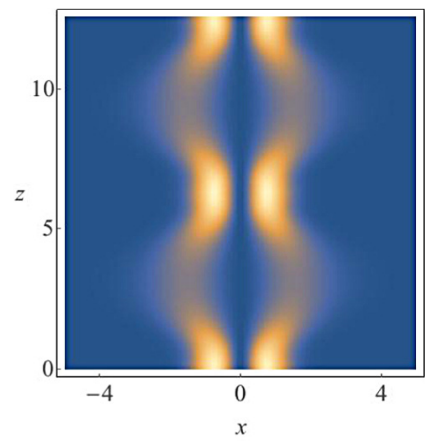

(a) $|\zeta|=0.25$.

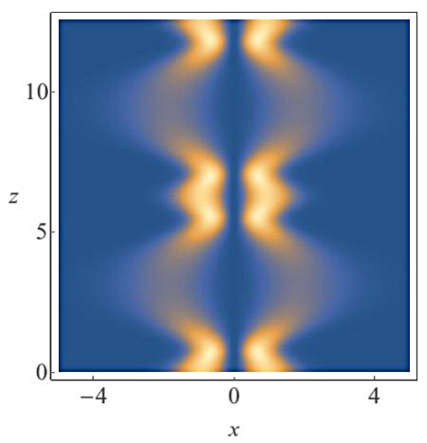

(b) $|\zeta|=0.5$.

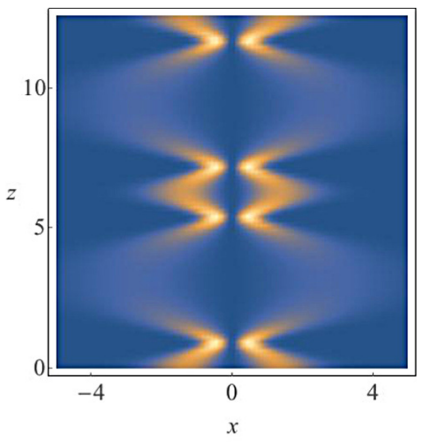

(c) $|\zeta|=0.75$

Fig. 10. Longitudinal plane $y=0$ of the optical intensity patterns for the $S U(1,1)$ Perelomov coherent states with $k=1$, $\varphi=\frac{\pi}{2}, \Omega=0.5 / z_{R}$ and different values of $|\zeta|$. Consistently with Fig. 8(c), in this plane the distributions present only two oscillating peaks. The oscillation amplitude becomes larger for larger values of $|\zeta|$. The variables $x$ and $z$ are measured in units of $w_{0}$ and $z_{R}$ respectively.

with $\left\langle\mathcal{K}_{0}\right\rangle_{0}={ }_{\mathcal{K}}\left\langle k, k\left|\mathcal{K}_{0}\right| k, k\right\rangle_{\mathcal{K}}=k[32,50]$. Thus

$$
\Delta \mathcal{K}_{1} \Delta \mathcal{K}_{2}=\frac{1}{2}\left[\left(\frac{1+|\zeta|^{2}}{1-|\zeta|^{2}}\right)^{2}+\left(\frac{4 \operatorname{Re}(\zeta) \operatorname{Im}(\zeta)}{\left(1-|\zeta|^{2}\right)^{2}}\right)^{2}\right]^{\frac{1}{2}}\left\langle\mathcal{K}_{0}\right\rangle_{0}
$$

As

$$
\left\langle\mathcal{K}_{0}\right\rangle_{P}^{k}=\frac{1+|\zeta|^{2}}{1-|\zeta|^{2}}\left\langle\mathcal{K}_{0}\right\rangle_{0}
$$

the inequality (51) is minimized whenever $\operatorname{Re}(\zeta)=0$ or $\operatorname{Im}(\zeta)=0$, i.e., either for $|\zeta|=0$ and all values of $\varphi$, or for $\varphi=\frac{s \pi}{2}, s=0,1, \ldots$, and all values of $|\zeta|$. For some other points $\zeta$ one might expect squeezing. This occurs if the variance of one of the quadratures becomes smaller than the corresponding average uncertainty $\frac{1}{2}\left|\left\langle\mathcal{K}_{0}\right\rangle_{P}^{k}\right|$ at the expense of the other [51] (see also [32])

$$
\left(\Delta \mathcal{K}_{1}\right)^{2}<\frac{1}{2}\left\langle\mathcal{K}_{0}\right\rangle_{P}^{k}, \quad \text { or } \quad\left(\Delta \mathcal{K}_{2}\right)^{2}<\frac{1}{2}\left\langle\mathcal{K}_{0}\right\rangle_{P}^{k}
$$

In Fig. 11 we present the plots of $\left(\Delta \mathcal{K}_{1}\right)^{2}$ (red), $\left(\Delta \mathcal{K}_{2}\right)^{2}$ (blue) and $\frac{1}{2}\left\langle\mathcal{K}_{0}\right\rangle_{P}^{k}$ (black) as functions of $\varphi$ for $|\zeta|=0.5$ and $k=4$. One can see the alternating zones of squeezing for the quadratures $\mathcal{K}_{1}$ and $\mathcal{K}_{2}$. Note also that there are some regions, around the points $\varphi=\frac{\pi}{4}, \frac{3 \pi}{4}, \ldots$, for which the variances of both quadratures are larger than $\frac{1}{2}\left\langle\mathcal{K}_{0}\right\rangle_{P}^{k}$. Therefore, no squeezing exists at those points. The size of these regions increases for larger values of $|\zeta|$.

\section{The propagation factor}

The action of the ladder operators $A^{-}$and $B^{+}$on the Perelomov coherent states is given by

$$
A^{-}|\xi\rangle_{P}^{k}=\sqrt{\frac{2 k}{1-|\zeta|^{2}}} \zeta|\xi\rangle_{P}^{k+\frac{1}{2}}, \quad B^{+}|\xi\rangle_{P}^{k}=\sqrt{\frac{2 k}{1-|\zeta|^{2}}}|\xi\rangle_{P}^{k+\frac{1}{2}}
$$

The propagation factor for the $S U(1,1)$ Perelomov coherent states can be evaluated from (53)-(54) and (64). After some calculations we find

$$
M^{2}=2 k \frac{1+|\zeta|^{2}}{1-|\zeta|^{2}} \geq 1
$$




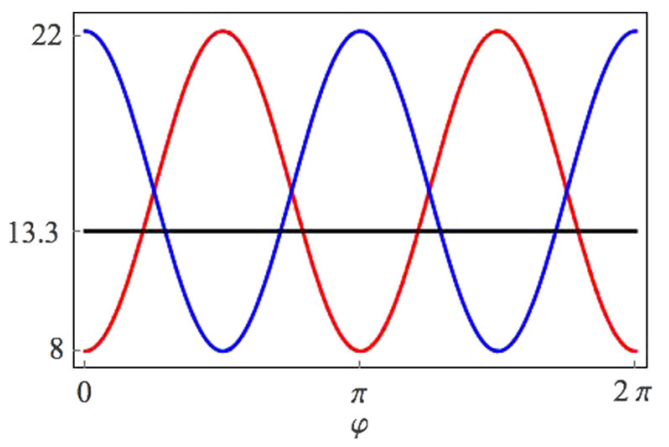

Fig. 11. (Color online) The variances $\left(\Delta \mathcal{K}_{1}\right)^{2}$ (red) and $\left(\Delta \mathcal{K}_{2}\right)^{2}$ (blue), for $|\zeta|=0.5$ and $k=4$, as functions of $\varphi$. The horizontal (black) line represents the (constant) value of the corresponding average uncertainty.

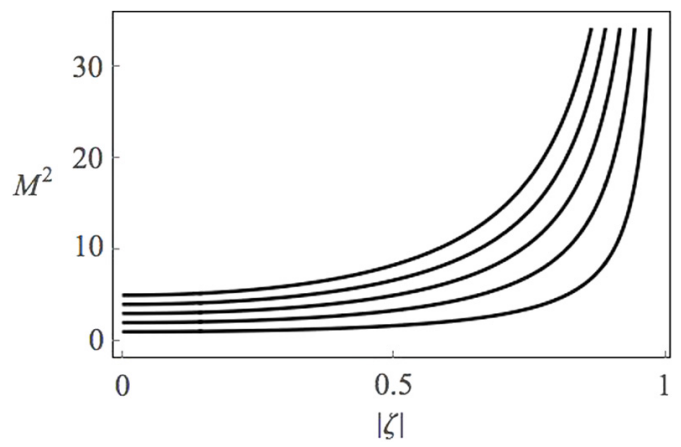

Fig. 12. Quality factor $M^{2}$ of the Perelomov coherent states for $k=\frac{1}{2}, 1, \frac{3}{2}, 2, \frac{5}{2}$ (lower curves correspond to smaller values of $k)$. It can be seen that, as $|\zeta| \rightarrow 0, M^{2} \sim 2 k$, and it tends to infinity as $|\zeta| \rightarrow 1$.

Note that the equality is satisfied only for the fundamental Gaussian mode characterized by $|\zeta|=0$ and $k=\frac{1}{2}$. As in the case of the Barut-Girardello coherent states we find $M^{2} \sim 2 k$ as $\zeta \rightarrow 0$. Besides, $M^{2}$ diverges as $|\zeta| \rightarrow 1$. In Fig. 12 it is shown the propagation factor (78) as a function of $|\zeta|$. From this figure we can conclude that the beam has a good quality for $|\zeta| \lesssim 0.5$, where $M^{2}$ is nearly constant. For larger values of $|\zeta|$ the beam is expected to exhibit a marked non-Gaussian behavior.

\subsection{Mode fields with definite total index number $n+m$}

In order to establish the intertwining algebra of the total index mode hierarchies, consider the set of operators

$$
\mathcal{J}_{+}=A^{+} B^{-}, \quad \mathcal{J}_{-}=A^{-} B^{+}, \quad \mathcal{J}_{0}=\frac{1}{2}\left(N_{x}-N_{y}\right) .
$$

It is straightforward to show that they satisfy the commutation relations of the $s u(2)$ algebra

$$
\left[\mathcal{J}_{-}, \mathcal{J}_{+}\right]=-2 \mathcal{J}_{0}, \quad\left[\mathcal{J}_{0}, \mathcal{J}_{ \pm}\right]= \pm \mathcal{J}_{ \pm} .
$$

The operators $\mathcal{J}_{ \pm}$raise one of the labels of the HG mode and lower the other one (see Fig. 13). Thus, the HG modes are transformed in such a way that the total index number $n+m$ is left invariant. With this in mind, let us define the parameters $j=\frac{1}{2}(n+m)$ and $v=\frac{1}{2}(n-m)$. We say that the operators $\mathcal{J}_{ \pm}$intertwine modes of the same $j$-hierarchy. The complete mode space splits into the direct sum of the set of subspaces $\left\{\mathcal{H}^{j}, j=0, \frac{1}{2}, 1, \ldots\right\}$, which are the spans of each particular $j$-hierarchy and 


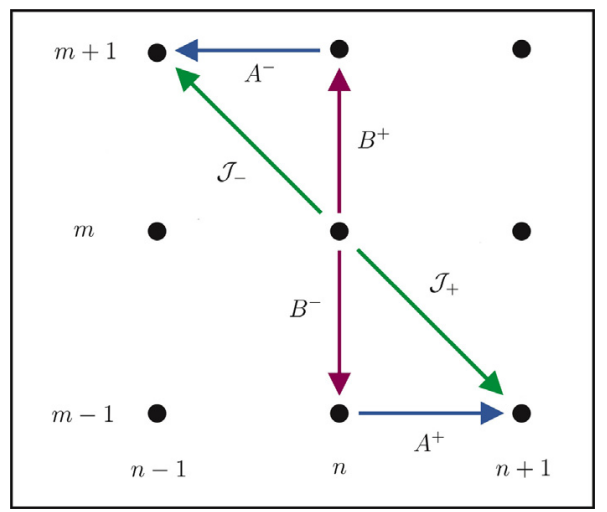

Fig. 13. Action of the operators $\mathcal{J}^{ \pm}$on the Hermite-Gaussian modes.

correspond to different irreducible representations of $S U(2)$. Also $n=j+v, m=j-v=2 j-n \geq 0$, $n=0,1, \ldots, 2 j$, and hence $v=n-j=-j,-j+1, \ldots, j-1, j$.

Now, by fixing the vector $|j, v\rangle_{\mathcal{J}}$ in such a way that $|n, m\rangle=|j+v, j-v\rangle=|j, v\rangle_{\mathcal{J}}$, the action of the operators $\mathcal{J}_{ \pm}, \mathcal{J}_{0}$ reads

$$
\begin{array}{rlrl}
\mathcal{J}_{+}|j, v\rangle_{\mathcal{J}} & =\sqrt{(j-v)(j+v+1)}|j, v+1\rangle_{\mathcal{J}}, & & \mathcal{J}_{+}|j, j\rangle_{\mathcal{J}}=0, \\
\mathcal{J}_{-}|j, v\rangle_{\mathcal{J}} & =\sqrt{(j+v)(j-v+1)}|j, v-1\rangle_{\mathcal{J}}, & & \mathcal{J}_{-}|j,-j\rangle_{\mathcal{J}}=0, \\
\mathcal{J}_{0}|j, v\rangle_{\mathcal{J}} & =v|j, v\rangle_{\mathcal{J}} . &
\end{array}
$$

\section{5. $S U(2)$ Perelomov coherent states}

The Perelomov approach for constructing generalized coherent states may now be applied to these hierarchies. By using the mode $|j,-j\rangle_{\mathcal{J}}$ as the extremal state one has

$$
|\xi\rangle_{P}^{j}=D(\xi)|j,-j\rangle_{\mathcal{J}}, \quad \text { with } \quad D(\xi)=e^{\xi \mathcal{J}_{+}-\bar{\xi} \mathcal{J}_{-}} .
$$

Iterating the action of $\mathcal{J}_{+}$on the extremal state we get

$$
\left(\mathcal{J}_{+}\right)^{s}|j,-j\rangle_{\mathcal{J}}=\sqrt{\frac{s !(2 j) !}{(2 j-s) !}}|j,-j+s\rangle_{\mathcal{J}}, \quad \text { for } \quad s \leq 2 j .
$$

Then, by using the disentangling formula

$$
D(\xi)=e^{\zeta \mathcal{J}_{+}} e^{\ln \left(1+|\zeta|^{2}\right) \mathcal{J}_{0}} e^{-\bar{\zeta} \mathcal{J}_{-}}, \quad \zeta=\frac{\xi}{|\xi|} \tan |\xi|,
$$

we arrive at

$$
|\xi\rangle_{P}^{j}=\frac{\zeta^{j} \sqrt{(2 j) !}}{\left(1+|\zeta|^{2}\right)^{j}} \sum_{\nu=-j}^{j} \frac{\zeta^{\nu}}{\sqrt{(j+v) !(j-v) !}}|j, v\rangle_{\mathcal{J}} .
$$

In the position representation the corresponding mode amplitude reads

$$
U_{P}^{j}(\mathbf{r}, z)=\langle\mathbf{r} \mid \xi\rangle_{P}^{j}=\frac{\zeta^{j} \sqrt{(2 j) !}}{\left(1+|\zeta|^{2}\right)^{j}} \sum_{\nu=-j}^{j} \frac{\zeta^{\nu}}{\sqrt{(j+v) !(j-v) !}} U_{j+v, j-v}(\mathbf{r}) .
$$




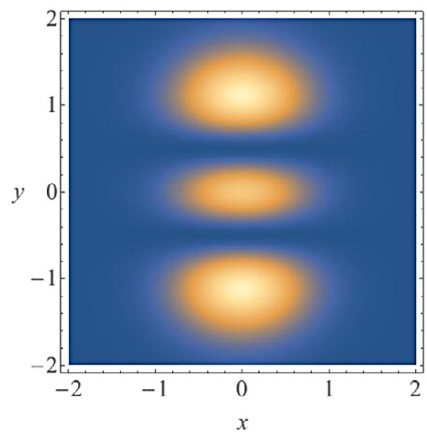

(a) $|\zeta|=0$.

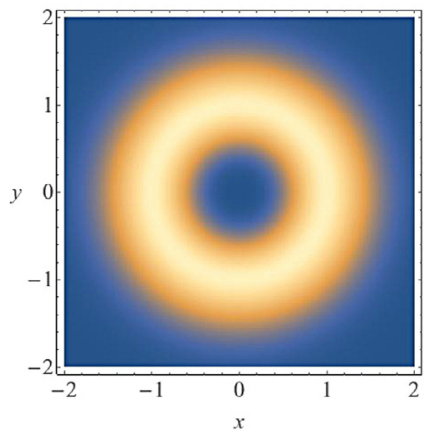

(b) $|\zeta|=1$.

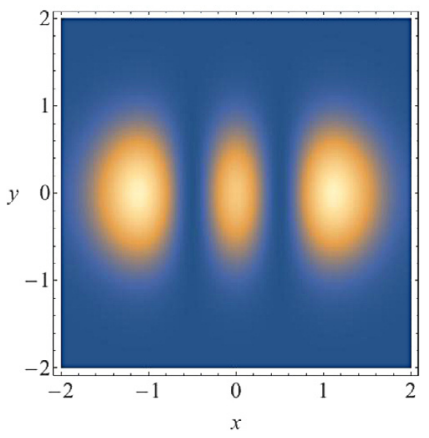

(c) $|\zeta|=100$.

Fig. 14. Intensity distributions of the Perelomov coherent states at the transversal plane $z=0$, for $j=1, \varphi=\frac{\pi}{2}$ and the indicated values of $|\zeta|$. In the case $|\zeta|=1$ the pattern exhibits axial symmetry due to the balanced contribution of the HG modes to the coherent state. The variables $x, y$ are measured in units of $w_{0}$.

In Fig. 14 we present the optical intensity carried by these modes at the transversal plane $z=0$, for $j=1, \varphi=\frac{\pi}{2}$ and different values of $|\zeta|$. The pattern consists of a set of $2 j+1$ maxima rotating around the origin as $|\zeta|$ increases. For the special value $\zeta=i\left(|\zeta|=1, \varphi=\frac{\pi}{2}\right)$ the beam exhibits axial symmetry due to the balanced contribution of the HG modes in the superposition (87). Fig. 15, on the other hand, shows the behavior of the optical intensity pattern as a function of $|\zeta|$ for $y=0, \varphi=\frac{\pi}{2}$, and different values of $j$. For this value of $y$ and integer values of $j$ the pattern presents only one peak in the region $|\zeta|<1$, consistently with Fig. 14(a). For $|\zeta|=1$, in all cases, the distribution shows two peaks (compare to Fig. 14(b)) that increase their distance for larger values of $j$. In the regime $|\zeta|>1$ the pattern exhibits the whole $2 j+1$ maxima due to its rotation around the optical axis. Finally, the evolution of these coherent states along the optical axis is presented in Fig. 16. In this figure is depicted the longitudinal plane $y=0$ for the choice $\Omega=\frac{0.5}{z_{R}}, j=3, \varphi=\frac{\pi}{2}$, and $|\zeta|=0,1,100$. Note the correspondence between these plots and that of Fig. $15(\mathrm{c})$ for different values of $|\zeta|$.

\section{Variances and squeezing}

The commutation relations (80) imply the inequality

$$
\Delta \mathcal{J}_{1} \Delta \mathcal{J}_{2} \geq \frac{1}{2}\left|\left\langle\mathcal{J}_{0}\right\rangle\right|
$$

with the quadratures $\mathcal{J}_{1,2}$ defined by

$$
\mathcal{J}_{1}=\frac{1}{2}\left(\mathcal{J}_{+}+\mathcal{J}_{-}\right), \quad \mathcal{J}_{2}=\frac{1}{2 i}\left(\mathcal{J}_{+}-\mathcal{J}_{-}\right) .
$$

For the $S U(2)$ coherent modes we get

$$
\begin{aligned}
& \left(\Delta \mathcal{J}_{1}\right)^{2}=-\frac{1}{2}\left[1-\left(\frac{2 \operatorname{Re}(\zeta)}{1+|\zeta|^{2}}\right)^{2}\right]\left\langle\mathcal{J}_{0}\right\rangle_{0}, \\
& \left(\Delta \mathcal{J}_{2}\right)^{2}=-\frac{1}{2}\left[1-\left(\frac{2 \operatorname{Im}(\zeta)}{1+|\zeta|^{2}}\right)^{2}\right]\left\langle\mathcal{J}_{0}\right\rangle_{0} \\
& \left\langle\mathcal{J}_{0}\right\rangle_{P}^{j}=\frac{1-|\zeta|^{2}}{1+|\zeta|^{2}}\left\langle\mathcal{J}_{0}\right\rangle_{0}
\end{aligned}
$$




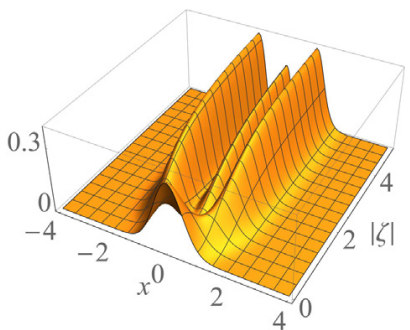

(a) $j=1$.

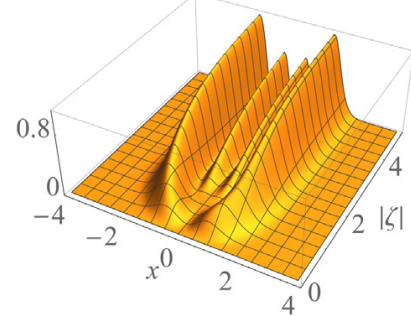

(b) $j=\frac{3}{2}$.

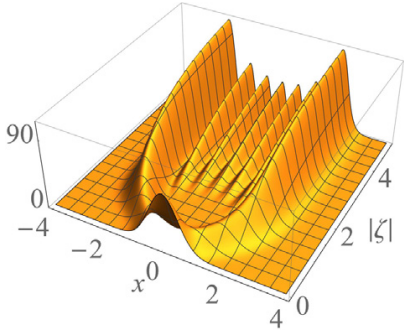

(c) $j=3$.

Fig. 15. Optical intensity of the $S U(2)$ Perelomov coherent states as functions of $|\zeta|$, for $y=0, \varphi=\frac{\pi}{2}$ and $j=1, \frac{3}{2}$, 3. The variable $x$ is measured in units of $w_{0}$.

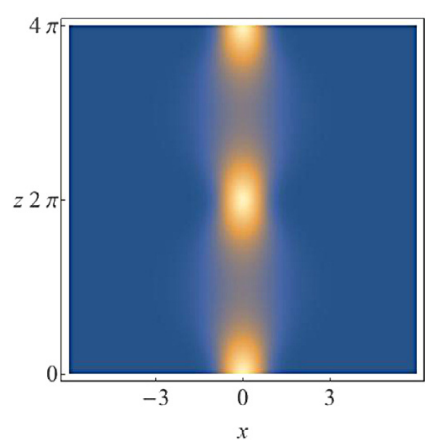

(a) $|\zeta|=0$.

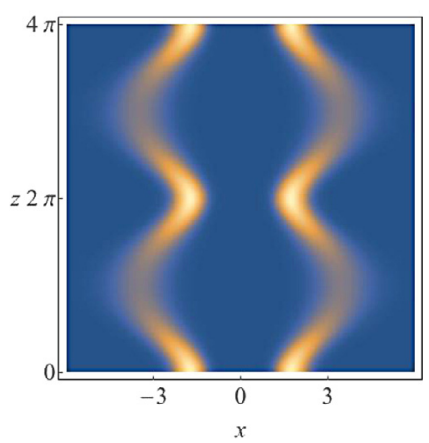

(b) $|\zeta|=1$.

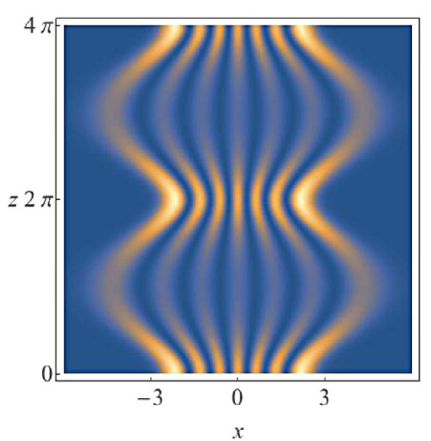

(c) $|\zeta|=100$.

Fig. 16. Longitudinal plane $y=0$ of the optical intensity $\left|U_{P}^{j}(\mathbf{r}, z)\right|^{2}$ with $\Omega=0.5 / z_{R}, j=3$ and $\varphi=\frac{\pi}{2}$, for the values of $|\zeta|$ indicated in each case. These patterns are consistent with those shown in Fig. 15(c). The coordinates $x$ and $z$ are measured in units of $w_{0}$ and $z_{R}$ respectively.

with $\left\langle\mathcal{J}_{0}\right\rangle_{0}={ }_{\mathcal{J}}\left\langle j,-j\left|\mathcal{J}_{0}\right| j,-j\right\rangle_{\mathcal{J}}=-j$. As

$$
\Delta \mathcal{J}_{1} \Delta \mathcal{J}_{2}=\frac{1}{2}\left[\left(\frac{1-|\zeta|^{2}}{1+|\zeta|^{2}}\right)^{2}-\left(\frac{4 \operatorname{Re}(\zeta) \operatorname{Im}(\zeta)}{\left(1+|\zeta|^{2}\right)^{2}}\right)^{2}\right]^{\frac{1}{2}}\left\langle\mathcal{J}_{0}\right\rangle_{0}
$$

the equality sign in (89) is valid whenever $\operatorname{Re}(\zeta)=0$ or $\operatorname{Im}(\zeta)=0$, i.e., either for $\zeta=0$ or for $\varphi=\frac{s \pi}{2}$, $s=0,1,2, \ldots$, and any value of $|\zeta|$. The squeezing of the quadratures $\mathcal{J}_{1}$ or $\mathcal{J}_{2}$ arises for the values of $\zeta$ that make the corresponding variance smaller than the average uncertainty $\frac{1}{2}\left|\left\langle\mathcal{J}_{0}\right\rangle_{P}^{j}\right|$. As it is shown in Fig. 17, this occurs around the points $\varphi=s \pi$ and $\varphi=(2 s+1) \frac{\pi}{2}, s=0,1,2, \ldots$, respectively. There are, however, some zones of the complex plane, in the vicinity of the points $\varphi=\frac{\pi}{4}, \frac{3 \pi}{4}, \ldots$, for which the variances of both quadratures are larger than the corresponding average uncertainty.

\section{The propagation factor}

In order to determine the variances of the canonical variables $\mathbf{r}, \mathbf{p}$ it is necessary to establish the action of the ladder operators $A^{ \pm}$and $B^{ \pm}$on $|\xi\rangle_{P}^{j}$. After some calculations we get

$$
A^{-}|\xi\rangle_{P}^{j}=\sqrt{\frac{2 j}{1+|\zeta|^{2}}} \zeta|\xi\rangle_{P}^{j-\frac{1}{2}}, \quad B^{-}|\xi\rangle_{P}^{j}=\sqrt{\frac{2 j}{1+|\zeta|^{2}}}|\xi\rangle_{P}^{j-\frac{1}{2}}
$$




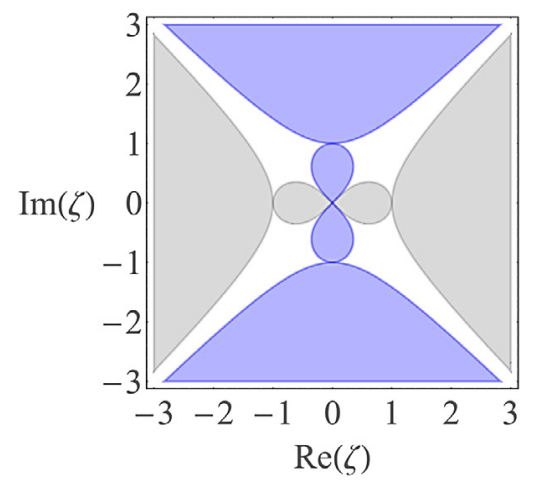

Fig. 17. (Color online) Regions of the complex plane where squeezing is expected, the gray zone corresponds to squeezing in $\mathcal{J}_{1}$ and the blue one to $\mathcal{J}_{2}$. The white zones correspond to points where the variances of both quadratures are larger than the average uncertainty.

The propagation factor (64) in this case turns out to be independent of $\zeta$, as it is reduced to

$$
M^{2}=2 j+1=n+m+1=\beta_{n m} .
$$

This last result coincides with the quality factor $M_{H G}^{2}$ of the HG mode $|n, m\rangle$.

\subsection{Comparison with the Glauber coherent states}

In the Glauber approach, it is possible to construct coherent states of a finite number of modes as the product of single mode ones [52] (see also [44]). In the case of two independent modes the coherent state is given by

$$
\left|\alpha_{x}, \alpha_{y}\right\rangle_{G}=\exp \left[-\frac{1}{2}\left(\left|\alpha_{x}\right|^{2}+\left|\alpha_{y}\right|^{2}\right)\right] \sum_{n, m=0}^{\infty} \frac{\alpha_{x}^{n} \alpha_{y}^{m}}{\sqrt{n ! m !}}|n, m\rangle, \quad \alpha_{x}, \alpha_{y} \in \mathbb{C} .
$$

In our case, it is straightforward to show that

$$
\Delta x=\Delta y=\frac{w}{2}, \quad \Delta p_{x}=\Delta p_{y}=\frac{w}{k_{0}}|S|, \quad \Delta x p_{x}=\Delta y p_{y}=\frac{w^{2}}{2 k_{0}} \operatorname{Re}(S),
$$

so that these states also minimize the Schrödinger-Robertson uncertainty relation on each degree of freedom:

$$
(\Delta x)^{2}\left(\Delta p_{x}\right)^{2}-\left(\Delta x p_{x}\right)^{2}=(\Delta y)^{2}\left(\Delta p_{y}\right)^{2}-\left(\Delta y p_{y}\right)^{2}=\frac{1}{4 k_{0}^{2}} .
$$

This fact leads to $M^{2}=1$ which indicates that the modes (97) are not affected by the propagation effects.

Remark that, as in the Glauber case, the generalized coherent states here constructed in the BarutGirardello approach, as eigenstates of the $s u(1,1)$ annihilator, also minimize the inequality involving the variances of the corresponding quadratures. This is no longer true for the Perelomov case, for which the inequality associated to the variances of the $s u(1,1)$ or $s u(2)$ generators is saturated only for some particular values of the parameters. It should be also noted that the Schrödinger-Robertson uncertainty relation involving the variances of the canonical variables, $\mathbf{r}$ and $\mathbf{p}$, is not minimized for any of the generalized coherent states but for the fundamental Gaussian mode. This means that diffraction effects are always expected to some extent. Yet, the generalized coherent states here discussed encode information about the algebraic structure of the mode space. The decomposition of this space into hierarchies unveils the symmetries of the system which are usually connected with dynamical variables that are of physical interest and allow the possibility of constructing different families of localized modes with a number of interesting properties. 


\section{Conclusions}

We have constructed the complete set of HG modes in a parabolic medium through the factorization method, by mapping the parabolic wave equation at a fixed transversal plane to a Schrödingertype equation for a two dimensional harmonic oscillator. The oscillating behavior of these modes as they propagate along the optical axis is encoded in the parameter $w(z)$ representing the beam width as a function of the longitudinal coordinate. We have also constructed $z$-dependent ladder operators $A^{ \pm}$, $B^{ \pm}$for the HG modes that generalize creation and annihilation operators for the harmonic oscillator, and new second order operators $\mathcal{K}_{ \pm}$and $\mathcal{J}_{ \pm}$that act as ladder operators in each infinite or finite dimensional hierarchy of the set of HG modes. Thus, we have shown that either the $s u(1,1)$ or the $s u(2)$ algebras generate the spectrum of propagation constants at any fixed transversal plane. A relevant point is that the index labeling the $S U(2)$ hierarchies is connected to the propagation constant $\beta_{n m}$. Hence, the corresponding subspaces are composed of modes with the same Gouy phase shift. We have applied the Barut-Girardello and Perelomov approaches to construct some families of coherent states, and determine the corresponding propagation factors. In the case of the coherent states associated to each $k$-hierarchy it was shown that the propagation factor $M^{2}$ behaves as $2 k$ for small values of the parameter $|\xi|$ labeling the coherent states. Therefore, a nearly Gaussian behavior is expected for moderate values of $k$. In the case of the $j$-hierarchies the propagation factor $M^{2}$ turns out to be the same as the propagation factor $M_{H G}^{2}$ of each of its HG components.

This group approach can be extended to paraxial mode space of different geometries. The generating algebras in each case lead to the classification of the mode basis into hierarchies associated to the symmetries of the system. Some results in this direction are in progress.

\section{Acknowledgments}

The authors acknowledge the financial support of CONACyT (Ph.D. scholarship for Z. Gress, grant 257292), Instituto Politécnico Nacional, Mexico (Project SIP20170233), the Spanish MINECO (Project MTM2014-57129-C2-1-P) and Junta de Castilla y León (VA057U16).

\section{References}

[1] A.E. Siegman, Lasers, University Science Books, California, 1986.

[2] T.P. Meyrath, F. Schreck, J.L. Hanssen, C.S. Chuu, M.G. Raizen, Opt. Express 13 (2005) 2843-2851.

[3] Y.K. Alp, O. Arkan, Digital Signal Processing 22 (2012) 1010-1023.

[4] S. Al-Awafi, S. Bougouffa, M. Babiker, Opt. Commun. 283 (2010) 1022-1025.

[5] A.P. Porfirev, R.V Skidanov, J. Opt. Technol. 82 (2015) 587-591.

[6] L. Novotny, E.J. Sánchez, X.S. Xie, Ultramicroscopy 71 (1998) 21-29.

[7] D.L. Andrews, Structured Light and its Applications: An Introduction to Phase-Structured Beams and Nano-Scale Optical Forces, Academic Press, London, 2008.

[8] A.V. Kovalev, V.V Kotlyar, S.G. Zaskanov, J. Opt. Soc. Am. A 31 (2014) 914-919,

[9] B. de Lima Bernardo, S. Azevedo, A. Rosas, Opt. Commun. 331 (2014) 194-197.

[10] A.S. Larkin, D.V. Pushkarev, S.A. Degtyarev, S.N. Khonina, A.B. Savelaev, Quantum Electron. 46 (2016) 733-737.

[11] S. Restuccia, D. Giovannini, G. Gibson, M. Padgett, Opt. Express 24 (2016) 27127-27136.

[12] G.V. Permitin, A.I. Smirnov, JETP 82 (1996) 395-402.

[13] H. Kogelnik, Appl. Opt. 4 (1965) 1562-1569.

[14] S. Choudhary, L.B. Felsen, Proc. IEEE 62 (1974) 1530-1541.

[15] M. Bornatici, O. Maj, Plasma Phys. Control. Fusion 45 (2003) 707-719.

[16] D. Gloge, D. Marcuse, J. Opt. Soc. Amer. 59 (1969) 1629-1631.

[17] M. Lax, W.H. Louisell, W.B. McKnight, Phys. Rev. A 11 (1975) 1365-1370.

[18] D. Stoler, J. Opt. Soc. Am. A 71 (1981) 334-341.

[19] S.J. van Enk, G. Nienhuis, Opt. Commun. 94 (1992) 147-158.

[20] R. Simon, N. Mukunda, J. Opt. Soc. Am. A 15 (1998) 2146-2155.

[21] G. Nienhuis, J. Visser, J. Opt. A: Pure Appl. Opt. 6 (2004) S248-S250.

[22] S.J.M. Habrakem, G. Nienhuis, J. Math. Phys. 51 (2010) 08270219 pages.

[23] S.G. Krivoshlykov, N.I. Petrov, I.N. Sissakian, Op. Quantum Electron. 18 (1986) 253-264.

[24] S.G. Krivoshlykov, N.I. Petrov, I.N. Sissakian, Sov. J. Quantum Electron. 16 (1985) 933-941.

[25] G. Nienhuis, L. Allen, Phys. Rev. A 48 (1993) 656.

[26] N.I. Petrov, Phys. Rev. A 90 (2014) 04381411 pages.

[27] L. Infeld, T.E. Hull, Rev. Modern Phys. 23 (1951) 21-68. 
[28] B. Mielnik, J. Math. Phys. 25 (1984) 3387-3389.

[29] A. Andrianov, N.V. Borisov, M.V. Ioffe, Theoret. Math. Phys. 61 (1984) 1078-1088.

[30] B. Mielnik, O. Rosas-Ortiz, J. Phys. A: Math. Gen. 37 (2004) 10007-10035.

[31] D.J. Fernández, J. Negro, M. Del Olmo, Ann. Phys. 252 (1996) 386-412.

[32] O. Rosas-Ortiz, S. Cruz y Cruz, M. Enríquez, Ann. Phys. 373 (2016) 346-373.

[33] R. Simon, N. Mukunda, Phys. Rev. Lett. 70 (1993) 880-883.

[34] V.P. Ermakov, Second Order Differential Equations. Conditions of Complete Integrability, Kiev University Izvestia, 1880 Series III 9125 (in Russian).

[35] O. Rosas-Ortiz, O. Castaños, D. Schuch, J. Phys. A 48 (2015) 445302.

[36] C. Brif, A. Mann, Quantum Semiclass. Opt. 9 (1997) 899-920.

[37] J. Guerrero, F.F. López-Ruiz, V. Aldaya, F. Cossío, J. Phys. A 44 (2011) 44530716 pages.

[38] O. Castaños, D. Schuch, O. Rosas-Ortiz, J. Phys. A 46 (2013) 075304.

[39] R.R. Puri, Phys. Rev. A 50 (1994) 5309-5316.

[40] P. Shanta, S. Chaturvedi, V. Srinivasan, G.S. Agarwal, C.L. Mehta, Phys. Rev. Lett. 72 (1994) 1147-1450.

[41] R.R. Puri, G.S. Agarwal, Phys. Rev. A 53 (1996) 1786-1790.

[42] I. Dhand, B.C. Sanders, H. de Guise, J. Math. Phys. 56 (2015) 11170520 pages.

[43] J. Schwinger, Quantum Theory of Angular Momentum, Academic Press, New York, 1965, pp. 229-279.

[44] A. Perelomov, Generalized Coherent States and their Applications, Springer-Verlag, Berlin, 1986.

[45] M. Abramowitz, I. Stegun, Handbook of Mathematical Functions with Formulas, Graphs, and Mathematical Tables, Dover, Washington DC, 1970.

[46] A.E. Siegman, SPIE 1224 (1990) 1-13.

[47] A.E. Siegman, SPIE 1868 (2004) 1-11.

[48] M.A. Banders, D. López-Mago, J.C. Gutiérrez-Vega, J. Opt. 12 (2010) 0657029 pages.

[49] Z. Mei, D. Zhao, J. Opt. Soc. Am. A 22 (2005) 1898-1902.

[50] C.C. Gerry, J. Kiefer, Phys. Rev. A 38 (1988) 191-196.

[51] K. Wodkiewicz, J.H. Eberly, J. Opt. Soc. Amer. B 2 (1985) 458-466.

[52] R. Glauber, Quantum Theory of Optical Coherence. Selected Papers and Lectures, Wiley-VCH Verlag GmbH \& Co, KGaA, Weinheim, 2007. 